\title{
DESIGN AND EXPERIMENTAL EVALUATION OF AN ELECTROSTATIC MICRO-GRIPPING SYSTEM
}

\author{
Defeng Lang, Marcel Tichem \\ $3 m E$, dep. PME, section PMA, Delft University of Technology
}

\begin{abstract}
The paper describes the modeling and experimental investigation of the application issues of electrostatic based micro-gripping. The design of an electrostatic gripping system for both grip force measure and pickup and place experiment is presented. A finite element model is made to study the gripping environment and process related features. The design and validation of the model are provided. Investigation of the influences that the gripping process gives out to and may receive from the operating environment is discussed. The preliminary result shows that grounded objects that come into the gripping area do not influence the gripping action significantly. Some real gripping actions are performed. Discussions on the scope of gripping environment and process related features are raised on basis of gripping experiments and observations. The phenomena of charging and discharging on the gripper isolator may add difficulties to gripping control. The investigation is concluded in a form of Process Data Sheet. The research draws an insight view on the application criteria of electrostatic gripping technology. Both advantages of the gripping principle e.g. flexible in terms of part dimension and geometry, and restrictions of the application are illustrated.
\end{abstract}

Key words: Micro-gripping; Electrostatic; Grip force; Modeling.

\section{INTRODUCTION}

The development trend of products miniaturization requests the assembly process to be extended from conventional scale into micro-scale. Microgripping, as the entire process which brings the downscaled parts from a loose state to a connected state, has different characteristics and raises new technological challenges compared to the conventional gripping process. Micro-gripping operations deal with parts with typical dimensions in the 
range of sub-millimetres up to a few millimetres; part features may be in the micron range. The typical accuracy in parts relation is in the range of 0.1 to $10.0 \mu \mathrm{m}$. Micro-objects react differently to forces compared to macroobjects. The surface related forces start to dominate over the volume related forces, which opens new possibilities to handle small objects. Electrostatic gripping is a method that is based on one of these possibilities.

A charge difference between two particles causes a force of repulsion or attraction. In electrostatic gripping this electric force field is used to operate target parts. The gripping method has been investigated and practically demonstrated by other researchers. An optically transparent bipolar gripper has been demonstrated ${ }^{1}$. Grip force higher than $2 \mathrm{mN}$ has been measured under 200 Volts bias voltage. The method is also implemented under a scanning electron microscope. By this means, the electrostatic field is generated by the microscope ${ }^{2}$. The tool can quickly pick up a 100 microns diameter metal ball.

However, to gain more understanding on the features of the gripping method is still very meaningful. This paper presents a computer model and test bench, which are made to investigate the gripping environment and process related features. Some results are addressed and presented in a form of the process data sheet ${ }^{3}$ of the operation method. The research provides more understanding on the electrostatic gripping principle; it also contributes to build up the knowledge of micro-gripping technology in general ${ }^{4}$.

\section{STRUCTURE OF THE GRIPPING SYSTEM}

A gripping and force measurement system has been developed which structure is shown in Figure 1 together with its equivalent circuit. The gripper is made as a cylinder with a diameter of $5.7 \mathrm{~mm}$. The material is aluminum. a piece of wire attached to its top. Via that different electrical potential can be added to the gripper. A piece of glass with thickness of 0.18 $\mathrm{mm}$ is used as the isolation layer, which is stuck to the bottom of the gripper by conductive glue. The gripper is attached to a robot. The two entities are electrically isolated from each other. The force measurement part includes a cantilever beam with well-defined spring constant, and a laser displacement sensor that measure the deflection of the cantilever. The smallest increment that can be measured (sensitivity = ration I think) of the force measurement is 8 micron Newtons. In the setup, the cantilever, the displacement sensor and the robot are all grounded and isolated from the gripper.

When enough potential difference is added between the gripper and the cantilever, the cantilever can be pulled up. The displacement sensor measures the deflection value, which is converted to force value. 

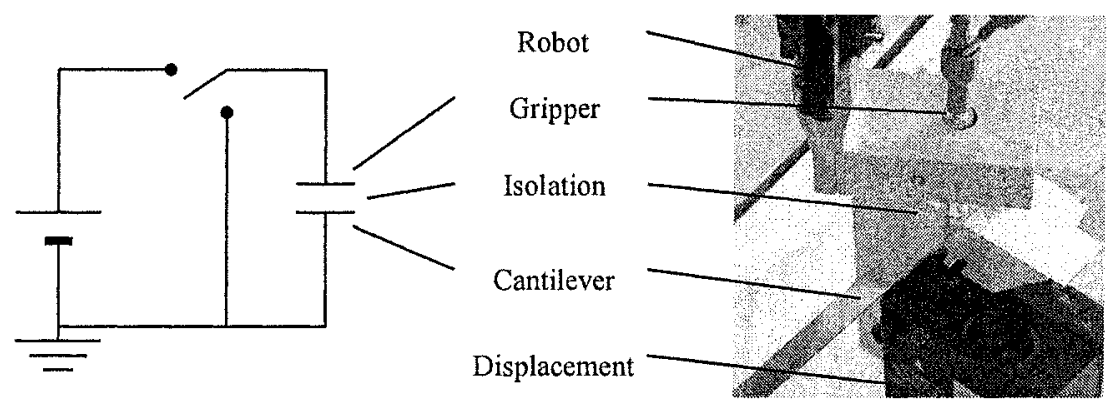

Figure 1. The structure of the gripping system and its equivalent circuit

\section{MODELING OF THE GRIPPING PROCESS}

A computer model is made on basis of the physical principle of the electrostatic interaction, which is used to study gripping environment related features.

\subsection{Electrostatic interaction}

By Coulomb's Law, Electrostatic force $F$ is defined as the electrical force of repulsion or attraction induced by an electric field $E$. The electrostatic force $F$ between two point charges $q$ at point $Q$ and $q^{\prime}$ at point $Q^{\prime}$ (with a distance $r$ between them) can be stated as in equation 1 . The constant $4 \pi$ is included because it simplifies some calculations. The $\varepsilon_{0}$, is the permittivity of free space.

$$
F=\frac{1}{4 \pi \varepsilon_{0}} \frac{q q^{\prime}}{r^{2}}
$$

Electrostatic forces in parallel plates can be described as in equation 2. Where $V$ is the applied voltage; $S$ is the cross-sectional area; $d$ is the separation distance:

$$
F_{z}=\frac{1}{2} \frac{\varepsilon_{0} \varepsilon S V^{2}}{d^{2}}
$$




\subsection{Finite element model}

To model the precise force interaction is hard and not the ultimate goal in this work. The simulation approach is to provide a good indication of the gripping process, and the acting force in the interaction. There are many parameters that influence the process in reality, what sometimes can lead to considerable difference from the simulation results. For simplification and to be able to verify, a model is made in Ansys emulating the measurement setup, which is shown in figure 2 .

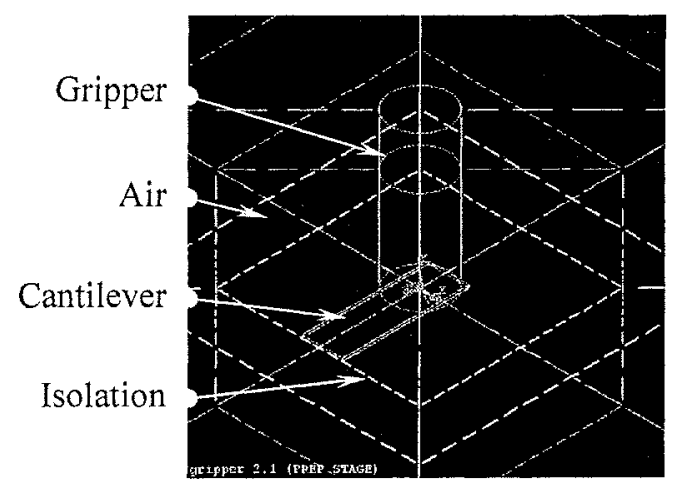

Figure 2. The frame structure of the gripping system model

Three different boundary conditions exist in the model. The first is the potential. The areas that form the gripper has to be assigned with a potential value, for instance 800 Volts; the areas that form the cantilever is set to 0 Volts. The second boundary condition is infinitive areas, which is applied to all the outer areas. The infinitive area is essential for this simulation to run correctly and produce good result. The third boundary condition is Maxwell surface, which is to calculate the nodal forces at the specified areas. After all the boundary conditions are set, the solution can be calculated.

\section{VALIDATON AND MODELING RESULTS}

To make use of the model, first the simulation results of interaction forces are compared with measuring value on the test bench. Further this model is applied to study the grip force under different geometries, voltages, and gripping situations. 


\subsection{Validation}

On the test bench, the force is measured and calculated by the deflection of the cantilever under certain potential difference and with certain distance away from the gripper.

First, to know the distance between the gripper and cantilever is crucial in this measurement. The gripper is moved away from the cantilever and the displacement is set to zero on the deflection sensor. This will be the point of reference. Then the gripper is moved in and brought closer to the cantilever; meanwhile 830 Volts is added to gripper. When the distance between the gripper and cantilever becomes small enough, the cantilever jumps towards the gripper and makes contact with isolation layer. The position of the gripper compared to the reference point is then known by reading the deflection. The gripper is then switched off and grounded.

Next, a smaller potential is set between the gripper and the cantilever. The cantilever will bend but not touch the gripper. From the difference between the position of the gripper and the reference point the air gap is known; from the displacement of the cantilever the force is calculated. With the distance of the air gap and the known potential, the force can be calculated and compared with the model.

Using the model, thirty different combinations of distance and potential where calculated. To make it easier to compare the model to the measurements, a graph is created and shown in figure 3. A power curve is fitted to the data. Two of the curves are shown in the graph and the corresponding equations are in the bottom left and bottom right of the graph area. The left equation corresponds to the 500 Volts curve; the right equation fits the 830 Volts curve.

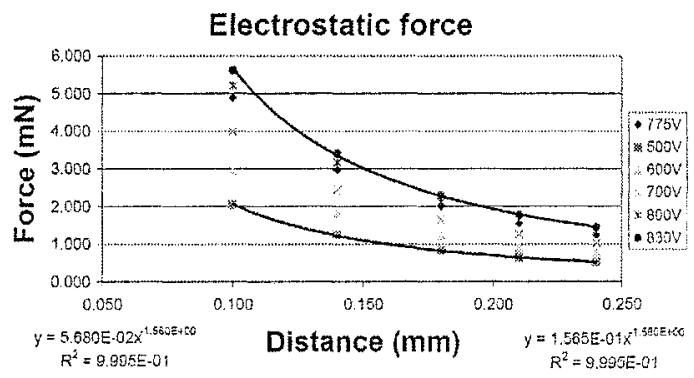

Figure 3. Graph of calculated forces

Figure 4 shows a diagram of a series of measurements, which were performed under potential differences of 500 Volts and 700 Volts. The 
vertical axis shows the electrostatic force, the horizontal axis shows the distance between the cantilever and the isolation layer. As shown by the figure, the predicted values correspond well to the measured values; although the measured electrostatic force is influenced by humidity of the air and the quality of the bonding with glue. This proves that the model is valid to be applied in further investigation.
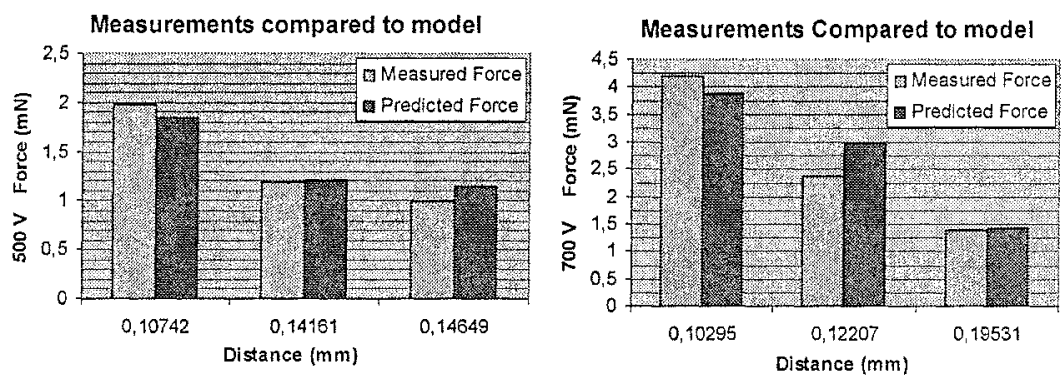

Figure 4. Measurements compared to model

\subsection{Prediction model of the gripping operation}

The argument has long existed as to whether the electrostatic gripping is reliable enough for industrial application. In more detail, the part is operated in an electrostatic field; any object that presents in this field may receive influence due to induction. Even the to be operated part has a risk of damage. Meanwhile, objects that come into this electrostatic field may give influence to the gripping operation. Thus it is interesting to know the magnitude of the influence.

The above evaluated model is modified to investigate the influence on the grip forces if a third object comes close to the gripper while it operates. Figure 5 shows the model. The part is a square shaped flat object with dimensions $8 * 8 * 0.5 \mathrm{~mm}$. The part is aligned under the centre of the gripper. The object which is inserted in the model to disturb the electric field is a cube with dimensions $5^{*} 5 * 5 \mathrm{~mm}$. The object is aligned with the part. The centre of the cube is positioned $10 \mathrm{~mm}$ above and $10 \mathrm{~mm}$ sideway from the centre of the bottom of the gripper. The boundary condition on the cube is set to 0 Volt (grounded). The gripper has a potential of 800 Volt compared to the part and the object. The air gap between the isolation layer and the part is set to $0.4 \mathrm{~mm}$. This is much larger than that occurs in reality. It should be noticed that the $Y$ direction is upward in the model.

The change of the potential field can be seen clearly by comparing the two graphs. The influence can be interpreted. The potential field becomes unsymmetrical around the gripper and underneath of the part. The change of 
the overall grip force is very limited in both perpendicular and horizontal direction.

Forces without object: $Y=5.86 \mathrm{E}-04 \mathrm{~N}, X=2.68 \mathrm{E}-07 \mathrm{~N}, Z=3.56 \mathrm{E}-08 \mathrm{~N}$.

Forces with object: $Y=5.82 \mathrm{E}-04 \mathrm{~N}, X=-5.31 \mathrm{E}-07 \mathrm{~N}, Z=-3.75 \mathrm{E}-09 \mathrm{~N}$.

The difference is within the range of the calculation errors that are inherent to the model. The simulation suggests that a grounded object in the vicinity of the gripper do not give large influence on the gripping contact.
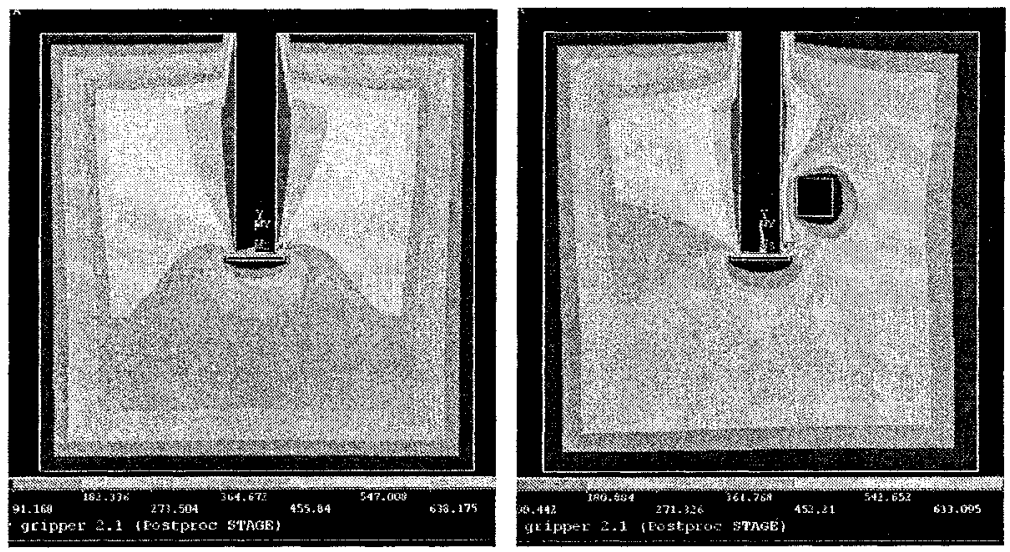

Figure 5. Potential field and forces without and with a grounded object

\section{EXPERIMENTS AND OBSERVATION}

Some pickup and place experiments were conducted to evaluate the actual performance of the gripping system. Parts made from different conductive and nonconductive materials were addressed. A test that was done on copper parts is presented here. The gripper used in this test has a diameter of 400 microns, isolated by 100 microns thick PTFE layer instead of glass; the target object was measured as 1500 microns diameter and 100 microns thick copper disk. Different voltages were set between the gripper and the part. To release the part, the strategy was to reverse charge the gripper. With 300 Volt added, the probability of pickup was $90 \%$ and probability of release was $100 \%$. With 200 Volt added, the probabilities were $35 \%$ and $57 \%$, respectively. With 150 Volt added, the probabilities were 0 . The gripper is able to pickup plastic and paper parts as well. The probabilities to pickup nonconductive or conductive parts are comparable, but to release a nonconductive part is more difficult. 
The charge accumulates over time on the gripper and on the other side of the isolation layer caused some phenomena during the experiments on the cantilever. The reasons behind some of these phenomena cannot be thoroughly explained at the current stage by the authors. Set the gripper and the cantilever away for some distance, the electrostatic force can keep on rising for few tens seconds if keep the electrical potential at constant. It suspects that charge is accumulating in this period, which results the force increasing.

The humidity of the operation environment gives large influence to the reaction of the cantilever in the electric field. Under a humid condition, humidity of the air over $60 \%$, the cantilever was seen to jump toward and contact the isolation layer then after about two seconds be released. The process can repeat itself with constant voltage added to the gripper. Few times under humidity below $40 \%$ the cantilever was attracted by the gripper and bumped on the isolation layer at the moment the voltage being switched off (gripper is set to grounded).

\section{PROCESS WINDOW OF ELECTROSTATIC GRIPPING}

According to the Experiments and observations, the electrostatic gripping can provide adequate force to pickup small objects. The dimension of the gripper is possible to be designed smaller than the target components, which is an advantage, means that it is more flexible in a micro-assembly environment. Meanwhile the same gripper can be applied to handle different parts; the flexibility concerning the part geometry is high.

However, comparing with other means of gripping, the grip force of this method is rather limited (for sure applying higher voltage is an option to raise the force, but it might hoist other limitations.). The Grip force may drop due to the gripped part having contact with conductive objects during the assembly process. In case that the gripping contact has to be assured while assembly takes place, the applicability of the principle can be questioned. Observing the formula of electrostatic force, a thinner isolation layer is beneficial. A gentle contact or a small gap is required in the handling to assure higher force while keeping the voltage low.

Further the gripping method can be concluded in a form of the Process Data Sheet PDS ${ }^{3}$. The purpose of PDS is to capturing the known data on issues related to micro-assembly processes. The data is considered to be important for training, education and engineering purposes in industry and universities. Main concerns of the electrostatic based gripping process are 
listed in table 1. The data captured in the PDS is based on a literature survey and on own research that addressed in this context.

Table 1. Process Data Sheet of Electrostatic based gripping

\begin{tabular}{|c|c|}
\hline Category & Description \\
\hline Process name & Electrostatic Based Gripping \\
\hline $\begin{array}{l}\text { Process } \\
\text { category }\end{array}$ & Gripping \\
\hline Description & $\begin{array}{l}\text { A charge difference between two particles causes a force of repulsion or } \\
\text { attraction. Electrostatic gripping is to utilize this force to operate target parts. } \\
\text { According to the number of electrodes, there are monopolar, bipolar grippers. }\end{array}$ \\
\hline \multirow[t]{4}{*}{$\begin{array}{l}\text { Process } \\
\text { window }\end{array}$} & $\begin{array}{l}\text { Flexible in terms of part geometry and dimension. Few tens microns till few } \\
\text { tens millimeters sized parts can be gripped. One contact surface is sufficient. } \\
\text { Grip force depends on the contact area between the gripper and part, } \\
\text { Permittivity of the isolation material and its thickness, the applied voltage } \\
\text { difference. }\end{array}$ \\
\hline & Typical value of the specific grip force is in the rang of $\mathrm{mN} / \mathrm{mm} 2$. \\
\hline & $\begin{array}{l}\text { Connection stiffness is limited. Grip force may drop during assembly process } \\
\text { due to having contact with conductive objects. }\end{array}$ \\
\hline & Part may have residue charge after being released. \\
\hline Cycle time & $\begin{array}{l}\text { The cycle time is reasonable short, in about sub-second to few seconds. The } \\
\text { time for charge accumulation and for residue charge dissipation is main } \\
\text { concern. }\end{array}$ \\
\hline Material type & $\begin{array}{l}\text { Both conductive and non-conductive material can be handled, but not } \\
\text { suitable for electric and magnetic sensitive materials and IC chips. }\end{array}$ \\
\hline $\begin{array}{l}\text { Environment } \\
\text { requirements }\end{array}$ & $\begin{array}{l}\text { Gripping action is not greatly influenced by surrounded conductive object if } \\
\text { grounded. Surrounded equipments may be affected due to the radiation. Low } \\
\text { humidity is preferred. }\end{array}$ \\
\hline
\end{tabular}

\section{CONCLUSION}

An electrostatic gripping system has been presented, which in one way is used to measure the grip force and another way is for real gripping operation. A computer model is built based on the gripping system. The model is verified by comparing the simulation result of the electrostatic force and the measuring result of the force value. Experiment of gripping small copper part shows the application possibility of this principle.

According to the simulation, influences given by a grounded object to the overall grip force is limited. Experiment and observation show that the principle is sensitive the humidity changing of the gripping environment. The phenomena of charging, discharging and residue charge on the gripper, isolator and part raise some uncertainty in the handling process. It gives extra difficulty to make the gripping process reliable and efficient. 
In further research, effort will be spent to further investigate how the gripping operation influences o the operation environment, and possibilities to reduce. A gripping system together operation strategy that uses lower electrical potential yet generates sufficient grip force will be the technological direction.

\section{ACKNOWLEDGMENT}

The authors wish to thank MSc. student H.J. Hendriks for his contribution to the experiment and modeling work, Mr. H.F.L. Jansen for building the experimental setup, and special thanks to Eng. F. Biganzoli for all the nice discussion and help.

\section{REFERENCES}

1. Eniko T. Enikov, Kalin V. Lazarov, An optically transparent gripper for micro assembly, Journal of Micromechatronics, Vol. 2, No. 2, pp. 121 140 (2004)

2. K. Tsuchiya, A. Murakami, G. Fortmann, M. Nakano, Y. Hatamura, Micro assembly and micro bonding in Nano Manufacturing World, SPIE Proceedings Vol. 3834 (Microrobotics and Microassembly) p.132 140, 1999

3. M. Tichem, F. Bourgeois, P. Lambert, V. Vandaele, D. Lang, Capturing Micro-Assembly Process Windows on Process Data Sheets, ISATP Proceeding, 2005

4. M. Tichem, D. Lang, B. Karpuschewski, A classification scheme for quantitative analysis of micro-grip principles, IPAS2003 Proceeding, p. 71-78, 2003 